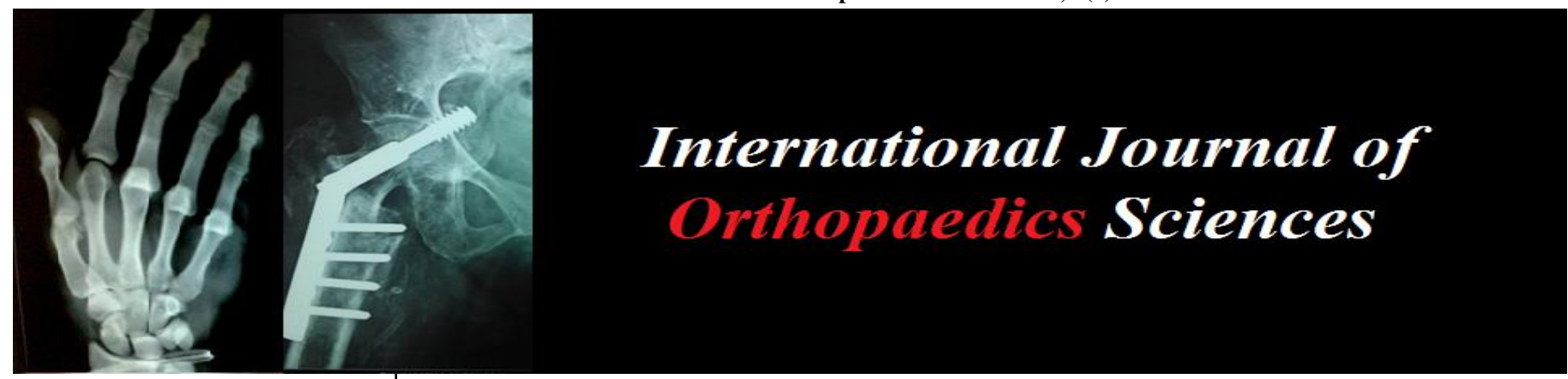

E-ISSN: 2395-1958

P-ISSN: 2706-6630

IJOS 2021; 7(2): 344-349

(C) 2021 IJOS

www.orthopaper.com

Received: 13-02-2021

Accepted: 15-03-2021

Dr. Shriniwas Vishnu Yadkikar Professor, Department of Orthopaedics PIMS(DU), Loni BK, Taluka - Rahata, Dist. Ahmednagar, Maharashtra, India

Dr. Vishnu S Yadkikar Former Professor \& Head, Department of Orthopaedics PIMS(DU), Loni BK, taluka Rahata, Dist. Ahmednagar, Maharashtra, India
Corresponding Author: Dr. Shriniwas Vishnu Yadkikar Professor, Department of Orthopaedics PIMS(DU), Loni BK, Taluka - Rahata, Dist. Ahmednagar, Maharashtra, India

\section{Role of Ilizarov ring fixator as a definitive treatment in management of compound comminuted paediatric tibia fractures}

\section{Dr. Shriniwas Vishnu Yadkikar and Dr. Vishnu S Yadkikar}

DOI: https://doi.org/10.22271/ortho.2021.v7.i2e.2650

\section{Abstract}

Introduction: Tibia shaft fractures are very common in children usually they are following fall. Generally they are very well treated with conservative management, but surgical intervention becomes mandatory in the presence of unstable, comminuted fractures with poor skin condition and compartment syndromes .

Material and Methods: There were 6 compound comminuted tibia fractures in between age group of 5 15 years. All were treated with ilizarov ring fixator application in department of orthopaedics of Pravara institute of medical science (DU) from April 2019 to January 2021.

Results: Early mobilization was initiated in all patients as per pain tolerance by post-operative day 2 or 3. Fracture united in all cases, no residual deformity seen in any case. Mild pintract infection was seen in 3 cases which were treated with regular dressings \& oral antibiotics. No delayed union, re fracture, limb length discrepancy was seen in any case, no bone grafting, corrective osteotomy was required in any case. All cases returned to their pre injury functional status. All cases completed their one year follow up. With little initial hesitation all patients well tolerated frame.

Conclusion: Ilizarov ring fixator can be considered as safe effective definitive treatment option for paediatric compound comminuted tibia fractures as its minimally invasive, biomechanically stable, dynamic, versatile treatment modality which promotes not only fracture union but also allows rapid early rehabilitation and functional recovery.

Keywords: ilizarov ring fixator, compound tibia fractures, paediatric fractures

\section{Introduction}

Tibia shaft fractures are very common in children usually they are following fall. Generally they are very well treated with conservative management, but surgical intervention becomes mandatory in the presence of unstable, comminuted fractures with poor skin condition and compartment syndromes, neurovascular injury.

Varity of treatment modalities are available for their treatment like Elastic nails, rigid nails, plates, external fixators. All of them are time tested and have their own merits and demerits. Elastic nails are one of the commonly used and preferred modality. Its minimally invasive but requires additional stability in the form of splints and immobilization. Incidence of nail migration are frequent, Non union, Limb length discrepancy may occur with elastic nails. Rigid nails cant be used in the presence of open physis. Plate fixation is also used but it may further damage the soft tissue cover. Risk of infection is always there with any form of internal fixation in the presence of compound fractures. In the presence of poor skin condition external fixators are preferred. External fixation is minimally invasive, suitable method and it's relatively easy. It can be done in the form of Mono lateral external fixation which is frequently used or with Ilizarov ring fixator or Taylors special frame or even by Supracutaneous platting. Once fracture union is achieved external fixator is removed \& there is no need for implant removal surgery as seen in internal fixation.

Withmonolateral external fixator early mobilization can be facilitated, wound care becomes easy as compared to internal fixation. But it doesn't permit weight bearing \& Malunion, residual deformity are frequently seen complications following its application.

Considering the pros\&cons associated with monolateral external fixator and other internal 
fixation methods, it was decided to use ilizarov ring fixator in management of compound comminuted paediatric fractures. Ilizarov ring fixator has a three dimensional versatile dynamic construct, which is biomechanically stable allowing wound care and permits full weight bearing during entire duration of treatment. Fracture management and dynamic deformity correction is simultaneously possible in this method. Fixator removal after fractureunionis easy. Even this method also has its own set of advantages \& disadvantages.

\section{Material \& Methods}

It was observational study carried out in department of orthopaedics of Pravara institute of medical science (DU) from April 2019 to January 2021 in which 6 compound \& comminuted paediatric tibia shaft fractures treated with Ilizarov ring fixator were studied. Approval from institutional ethical committee was taken for this study.

\section{Aims \& objectives}

To study role of ilizarov ring fixator as a definitive treatment in management of compound and comminuted paediatric tibia fractures. To study complications To study literature All cases were males between age group of 5 to 15 years. Road side accident was mechanism of injury in all cases. Amongst 6 cases 2 were Grade II and 4 were Grade III compound tibia fractures as per Gustilo Anderson classification. 2 patients had associated clavicle fractures.

\section{Inclusion criteria}

Compound and comminuted tibia shaft fractures in children between age group of 5 to 15 years

\section{Exclusion criteria}

Tibia fractures in children less than 5 years Pathological fractures Fractures with neurovascular injury After detailed clinical evaluation of patient standard AP \& Lateral x ray of affected limb were taken.

5 cases were having fractures with Meta- diaphyseal comminution and one was with purely distal metaphyseal fracture.

After through debridement of wound in operation theatre wound closure was done. In all cases primary skin closure was achieved no need for plastic surgery was required.

Average duration between admission and operation was one day.

Followed by closure standard Ilizarov 3 to 4140 degrees rings progressive construct was applied with help of $1.5-1.8 \mathrm{~mm}$ percutaneously passed trochar and olive wires under image intensifier control. Frame construct was made as per fracture pattern. All wires were juxtraphyesal \& no wire was passed through fracture site. Fracture compression was achieved by olive wires. Tension of around 100 to $110 \mathrm{~kg}$ was applied to wires. Fracture reduction was achieved by spanning it between rings. Ankle spanning was done in two cases. Excellent remodelling potential is seen in fractures of this age group but as per literature this potential reduces in more than 10 yrsof age, so emphasis was given to achieve near anatomical or anatomical reduction of fractures with the help of frame.

\section{Post operative Protocol}

IV Antibiotics were administered for 3 days post operatively. Regular dressing was done at periodic intervals. Active knee movements, straight leg raising was encouraged from post operative day one. As per pain tolerance in all patients full weight bearing with help of walker was started from post op day 2or 3. Parents were taught at home pintract dressings. Compression distraction cycle at the rate of $2 \mathrm{~mm}$ per day which was required in two cases 2 weeks. Average hospital stay was 10 days. X-rays were taken at 4 weeks interval.

\section{Results}

Wounds healed in all cases at around 2 weeks. Average fracture union time was 12 weeks $(14-16$ weeks $)$. Fracture union achieved in all cases. Mild pin tract infection was seen in 3 cases of grade III compound fractures which settled with oral antibiotics and regular dressings.

After 4 weeks active staircase climbing was also permitted. Follow up visits were made initially at 2 weeks interval till wounds healed and then every 4 weeks till fracture union.

Fracture union was assessed clinically by absence of tenders at fracture site and radiologically by callus formation in at least 3 cortices in AP and Lateral view.

Before frame removal all patients were made to walk with out support after disconnecting rods from rings, if patients were able to walk then again rings were connected and 3 weeks after that frame was removed. Frame removal was done after complete fracture union. Following frame removal posterior splint was applied for 2 weeks for protection.

In 2 cases of age less than 10 years fracture united with less than 5 degrees of deformity but it did not affect the functional outcome of patient.

All patients completed follow-up which were done at 3,6 and 12 months following fracture union. All cases with little initial hesitation well tolerated the frame all throughout treatment span. Post splint removal active knee ankle and foot mobilization was encouraged in all patients. All patients returned to their pre injury functional status.

No premature frame removal, Limb length discrepancy, osteomyelitis, compartment syndrome, delayed union, neurovascular injury was seen in any case No case required bone grafting or corrective osteotomy.

No refracture was seenin any case following fixator removal

Table 1: Distribution \& pattern of cases

\begin{tabular}{|c|c|c|c|c|c|c|c|}
\hline $\begin{array}{c}\text { Age in } \\
\text { years }\end{array}$ & $\begin{array}{l}\text { Number } \\
\text { Of cases }\end{array}$ & $\begin{array}{l}\text { Compound fracture type } \\
\text { As per Gustilo Anderson }\end{array}$ & Anatomical location & \begin{tabular}{|c|} 
Ankle spanning \\
Yes /No
\end{tabular} & $\begin{array}{l}\text { Pin tract } \\
\text { infection }\end{array}$ & \begin{tabular}{|c|} 
Residual deformity \\
In degrees
\end{tabular} & \begin{tabular}{|l|} 
Limb length \\
discrepancy
\end{tabular} \\
\hline 5 & 1 & II & Diaphysis & No & No & No & No \\
\hline 7 & 1 & II & Diaphysis & No & No & $<5$ recurvatum & No \\
\hline 9 & 1 & III & Diaphysis and comminuted & No & Yes & $<5$ recurvatum & No \\
\hline 9 & 1 & III & Diaphysis and comminuted & $\mathrm{NO}$ & Yes & No & No \\
\hline 11 & 1 & III & Distal metaphysis & Yes & Yes & No & No \\
\hline 15 & 1 & III & Comminuted meta diaphysis & Yes & No & No & No \\
\hline
\end{tabular}




\section{Discussion}

It was observational study in which 6 compound \& comminuted paediatric tibia shaft fractures between $5-15 \mathrm{yrs}$ of age treated with Ilizarov ring fixator were studied.

2 cases were grade II and 4 cases were grade III compound tibia fractures. As per pain tolerance early mobilization and weight bearing was started by pot op day 2 or 3 following frame application. Wounds healed in all cases at around 2 weeks. Average fracture union time was 12 weeks (14 16weeks). Fracture union achieved in all cases. No premature frame removal, Limb length discrepancy, osteomyelitis, compartment syndrome, delayed union; neurovascular injury was seen in any case. No refracture was seen any case following fixator removal. All patients returned to their pre injury functional status .No case required bone grafting or corrective osteotomy. All cases completed one year follow up. Low energy tibia fractures in children are often simple and can be treated with immobilization in cast ${ }^{[6,11,17]}$ however high energy fractures are associated with soft tissue injury so selection of treatment modality becomes difficult ${ }^{[1,11]}$ Unlike adults there are no clear cut guide lines for management of open tibial fractures in skeletally immature patient $[1,12,13]$

As per literature open fractures with more than $10 \mathrm{yrs}$ of age are associated with outcomes and complications like adults as compared to children less than $10 \mathrm{yrs}$ of age ${ }^{[1]}$. Remodelling potential decreases with age more than 10 years ${ }^{[1]}$ which may lead to residual deformity, therefore aim of treatment should be fracture union with minimal deformity in children more than 10 years ${ }^{[1,11]}$

Many treatment modalities are available from conservative to operative. Operative treatment ranges from elastic nails, rigid nails, plates, monolataeral external fixator \& ilizarov ring fixator, Taylors special frame, Supracutaneous platting $[2,4,5,6$, $11,17]$

Tibia has relatively poor soft tissue envelop \& variable blood supply which can be affected by initial trauma and further internal fixation may even reduce tissue viability leading to impairment in fracture healing ${ }^{[3,4]}$ Risk of infection can be more in open fractures fixed internally ${ }^{[4,7]}$

Elastic nails are minimally invasive and most favoured method by orthopaedic surgeons, but they are less stable in comminuted fractures ${ }^{[6,15]}$. Internal fixation by them also needs immobilization and support with splint for certain duration of time ${ }^{[15,16]}$

Internally fixed implant needs to be removed after certain duration this requires surgery which may lead to hypertrophic scar, wound breakdown if implant removal is delayed for considerable period of time then it becomes difficult as well and may need extensive surgery ${ }^{[3]}$

Mono lateral external fixator is one of the times tested method in presence of open wounds and unstable fractures ${ }^{[17]}$ it is easier to apply as compared to ring fixator ${ }^{[17]}$, but lack of rotational stability, Residualdeformity are some of the shortcomings associated with this method ${ }^{[1]}$ it also doesn't permit early full weight bearing unless its applied in ankle spanning manner ${ }^{[1,18]}$.

\section{Why we decided to use Ilizarov ring fixator ???}

Considering the pros \& cons associated with monolateral external fixator and other internal fixation methods, it was decided to use ilizarov ring fixator in management of compound comminuted paediatric fractures.

Ilizarov ring fixator has stable construct with rotational stability due to its three dimensional design ${ }^{[1,6]}$, it permits early weight bearing, dynamic deformity correction by means of gradual compression -distraction cycles [14] This also prevents occurrence of residual deformity ${ }^{[1,}{ }^{14]}$. Tensioned wires fixed with rings and rods provides stable construct with trampoline like effect which promotes axial micro motion of fractures fragments permitting union ${ }^{[1]}$. Early reduction \& stable fixation with ilizarov ring fixator is very useful \& reliable method of treatment for compound paediatric tibia fractures ${ }^{[1]}$. This method often proceeds to rapid union with few complications in comparison to other methods $[1,6]$ passing of juxtra physeal wires also doesn't disturb growth plate. Early mobilization and rehabilitation of patientspromotes early functional recovery ${ }^{[1,3]}$. Like every method it also has some advantages \& disadvantages such as

\section{Advantages of Ilizarov method}

Minimally invasive ${ }^{[1,3,6]}$ Biomechanically stable \& versatile construct ${ }^{[1]}$ Permits early mobilization, weight bearing on affected limb and rehabilitation [1, 3, 6] Dynamic method Permits residual deformity correction even after final frame application is done ${ }^{[1]}$.

Easy implant removal once fracture union is achieved

\section{Disadvantages}

Frame can be cumbersome Pin tract infection ${ }^{[1,6]}$ Difficulty may be encountered during clothing, sleeping, washing ${ }^{[6]}$ High learning curve Thus prior application of frame detailed counselling of parents regarding nature, method, duration of treatment and post operative care is a must.

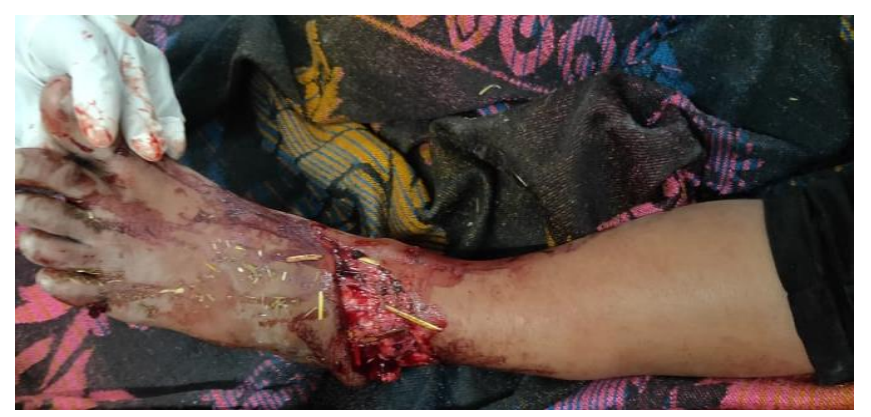

Fig 1: Pre operative clinical photo case 1

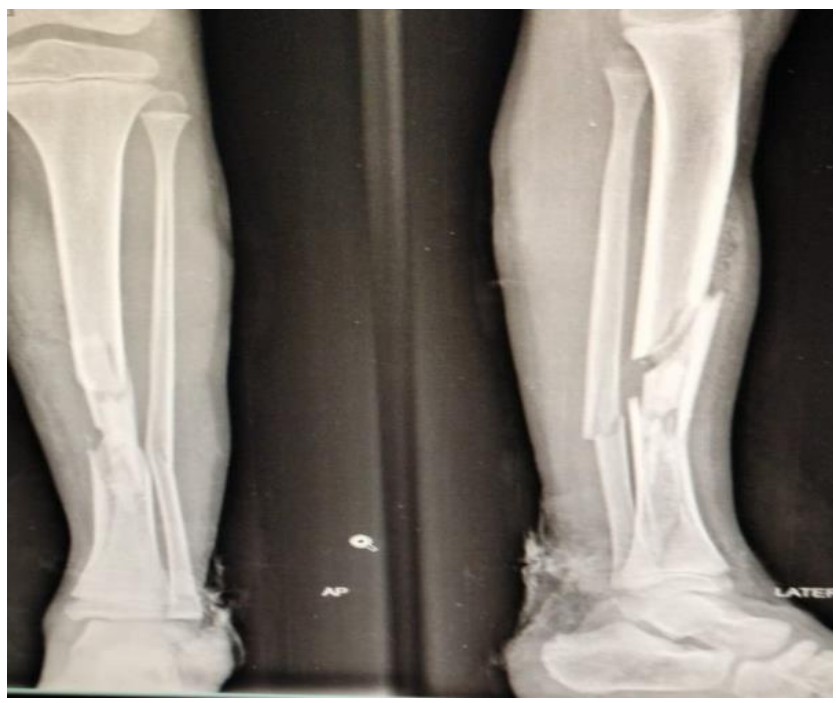

Fig 2: Pre-operative $\mathrm{X}$ ray Case 1 


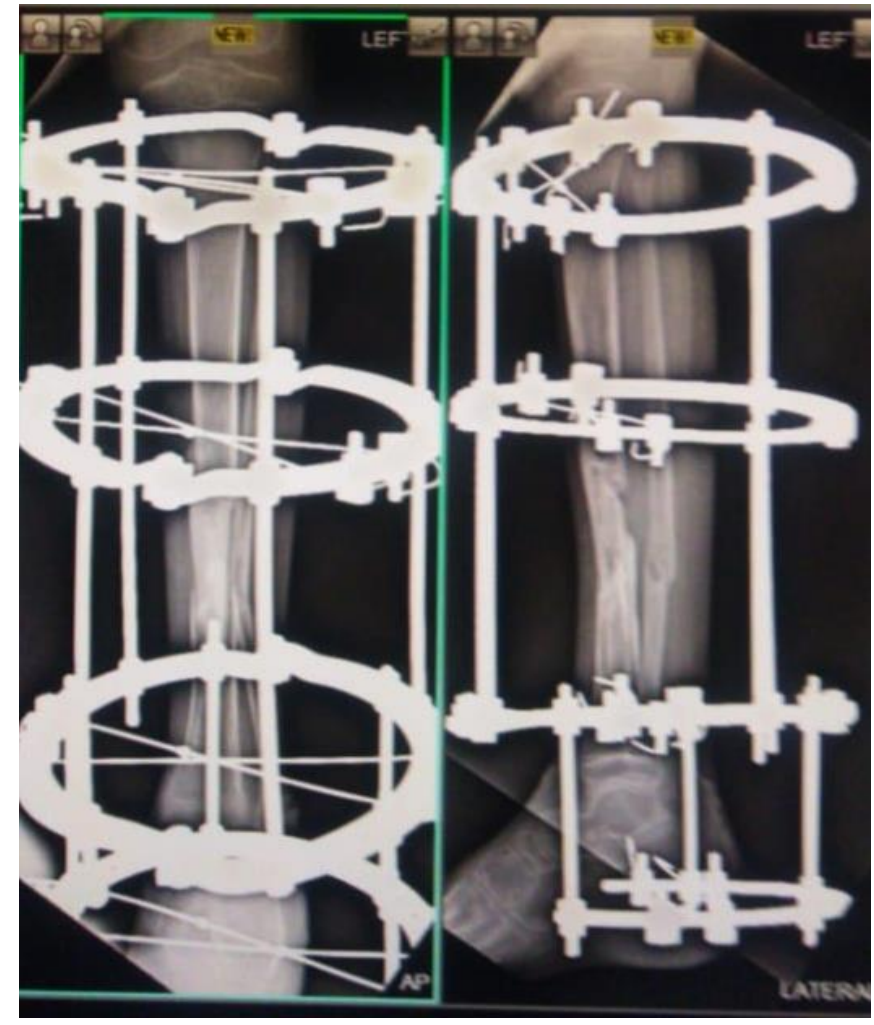

Fig 3: Post operative $\mathrm{x}$ ray case 1

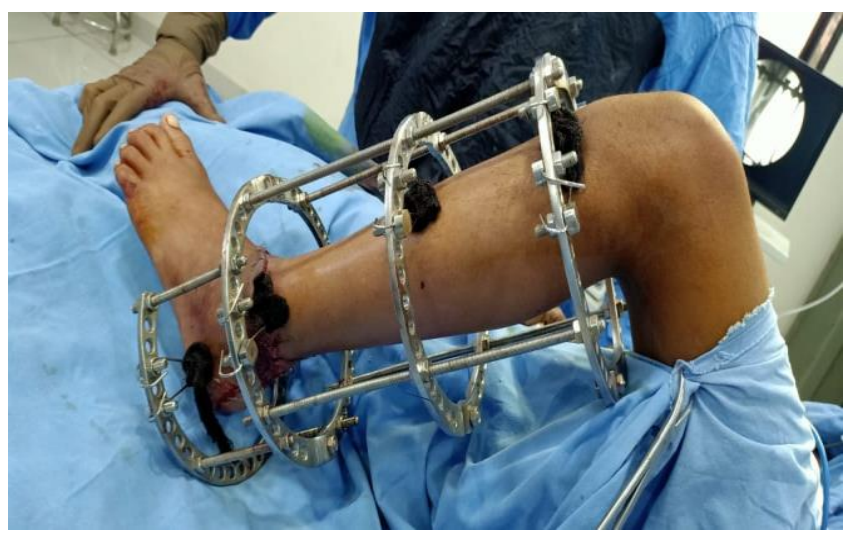

Fig 4: frame application case 1

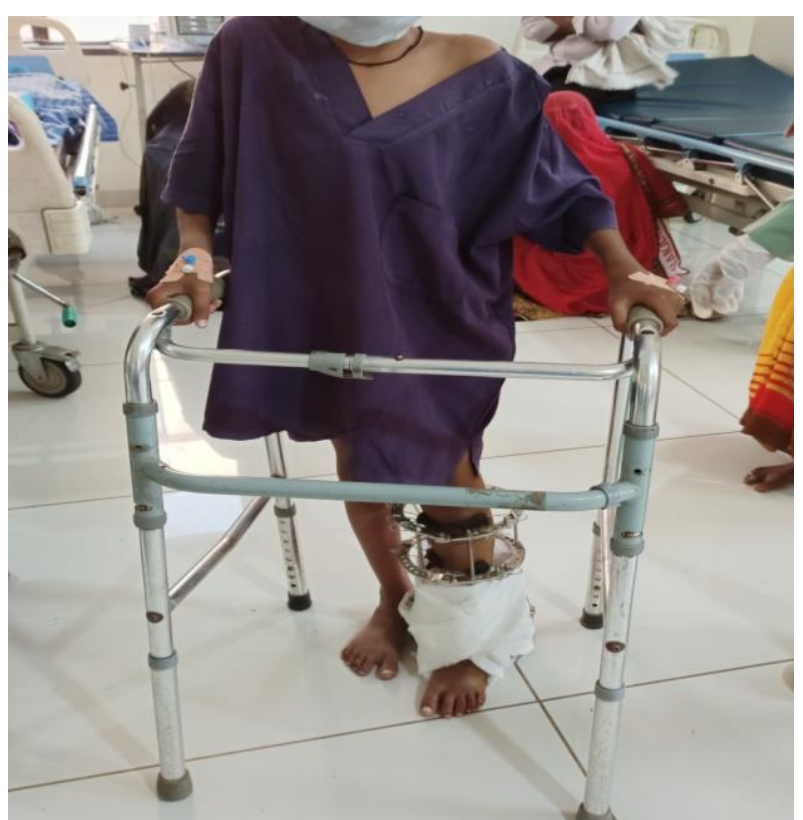

Fig 5: Early mobilization case 1

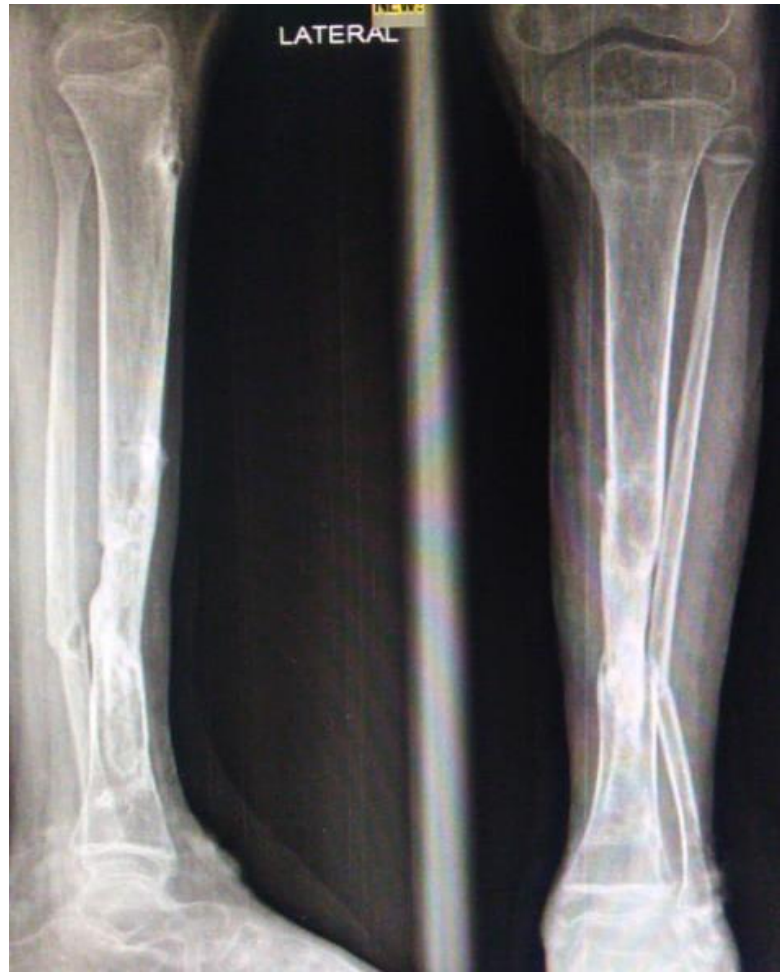

Fig 6: union $X$ ray case 1

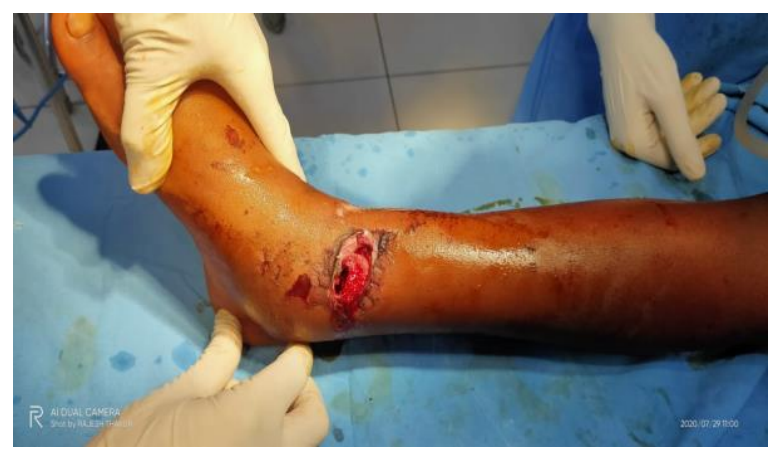

Fig 7: clinical photo Case 2

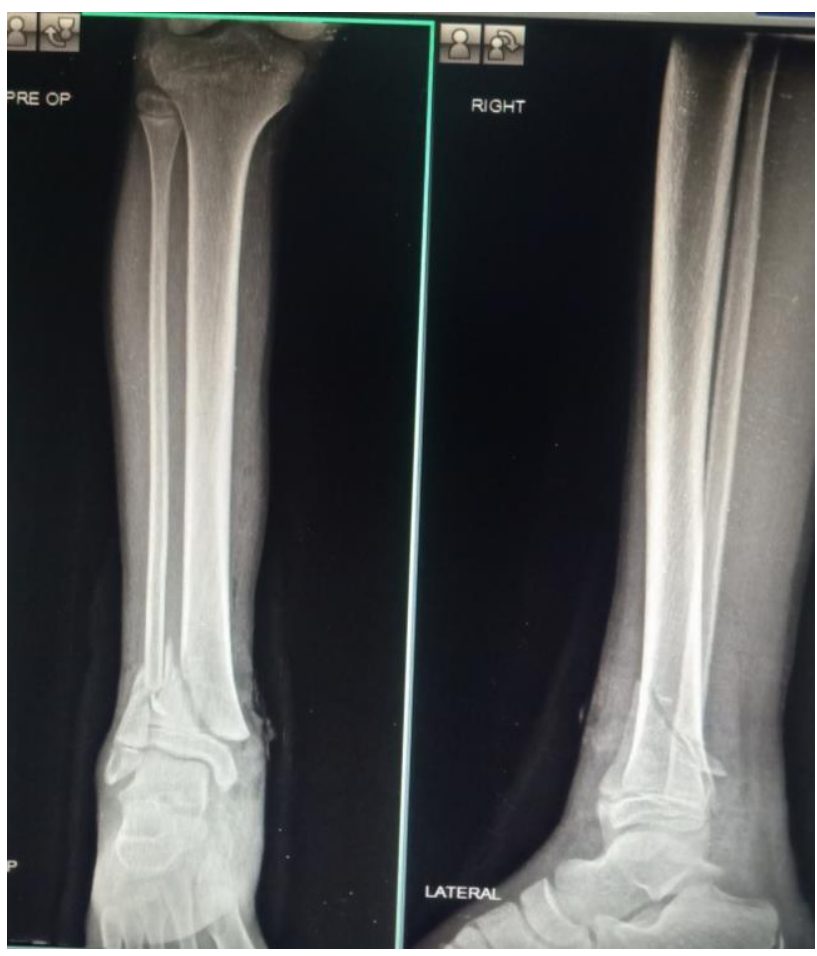

Fig 8: Pre operative $\mathrm{X}$ ray case 2 


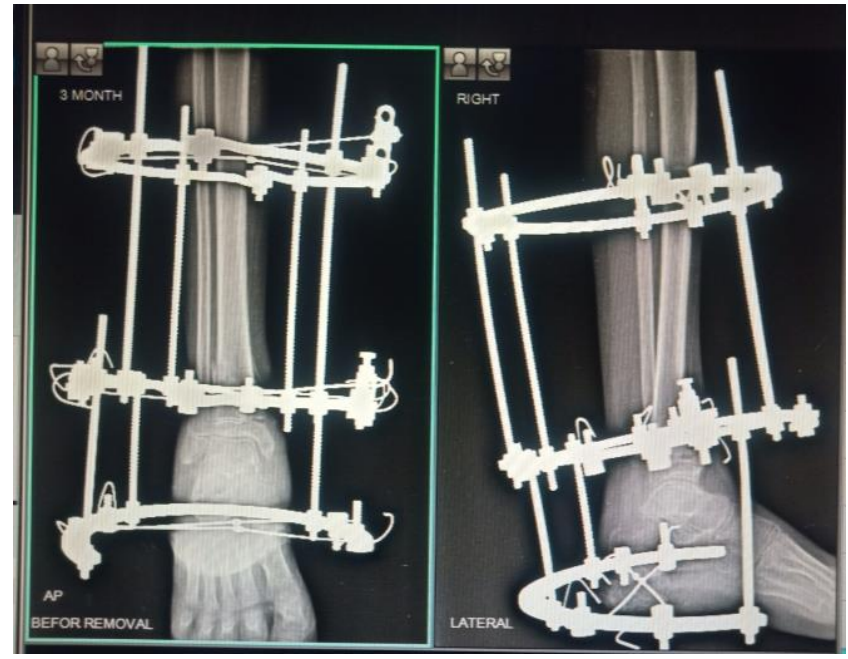

Fig 9: Post-operative $X$ ray case 2

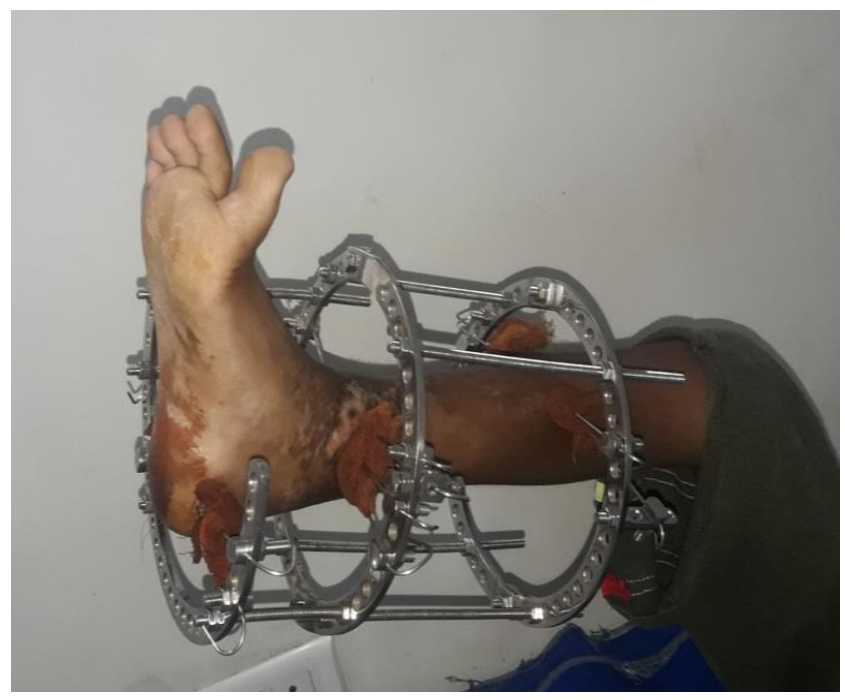

A.

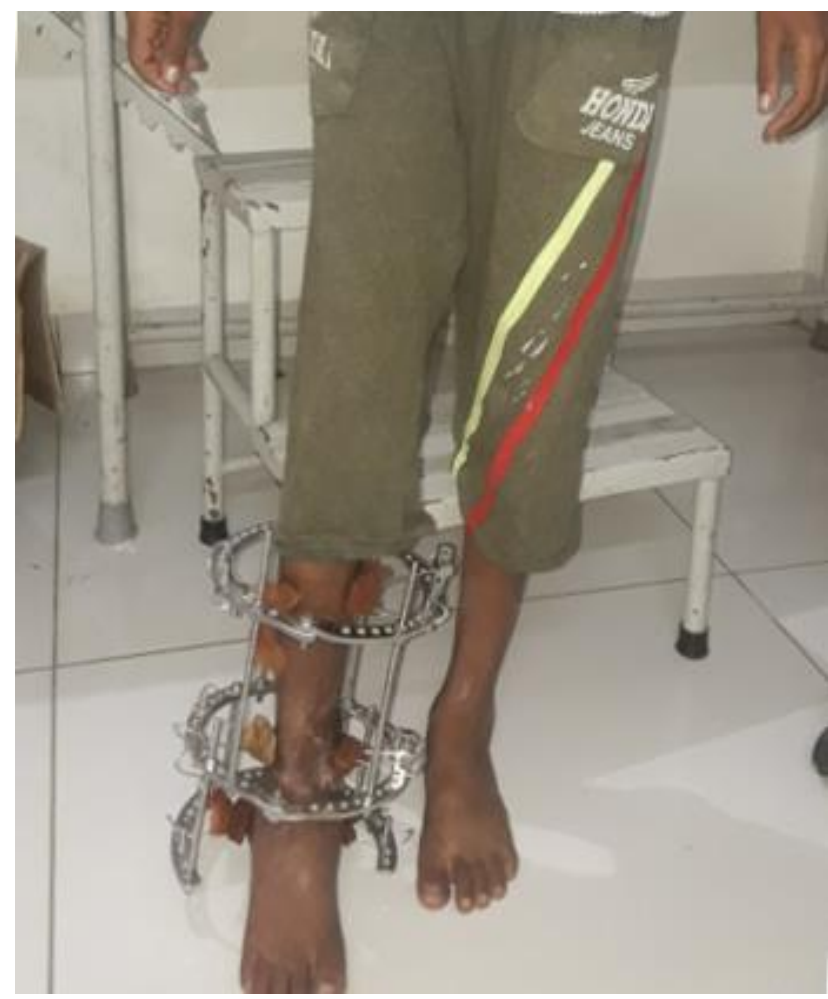

B.

Fig 10: Healed wound

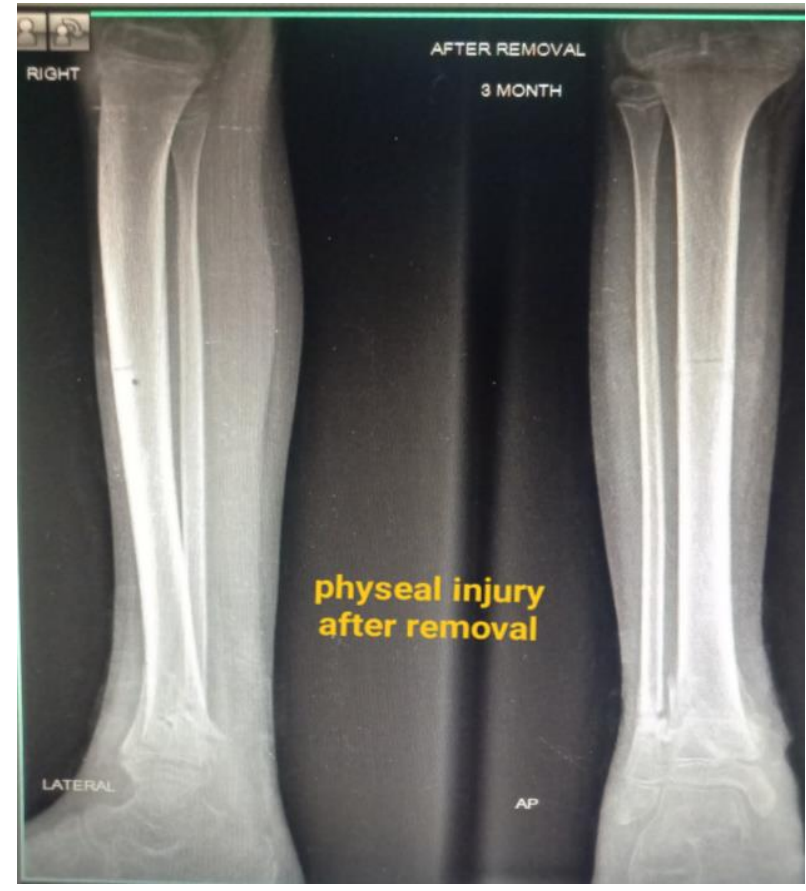

Fig 11: Union Xray Case 2

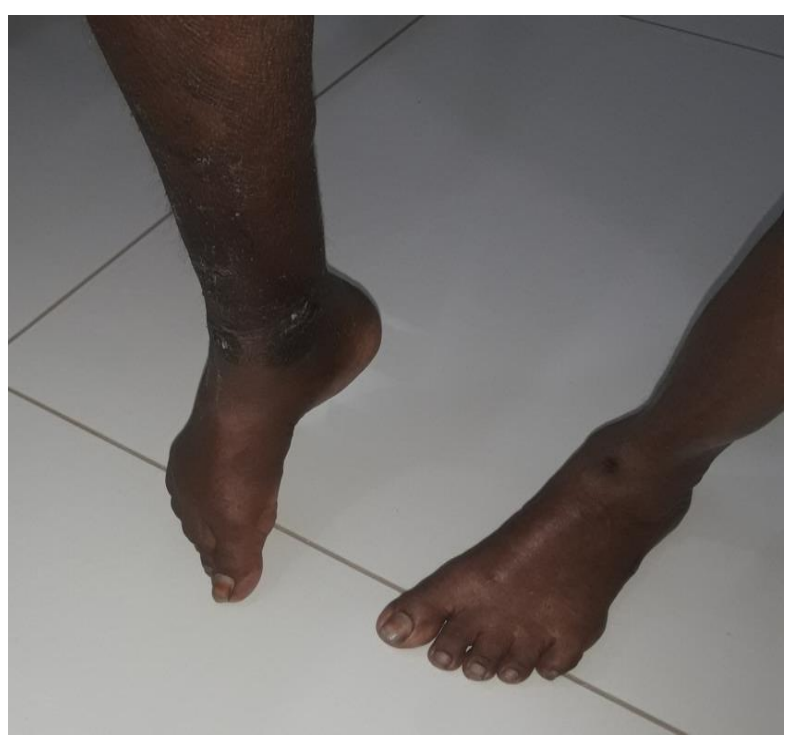

A.

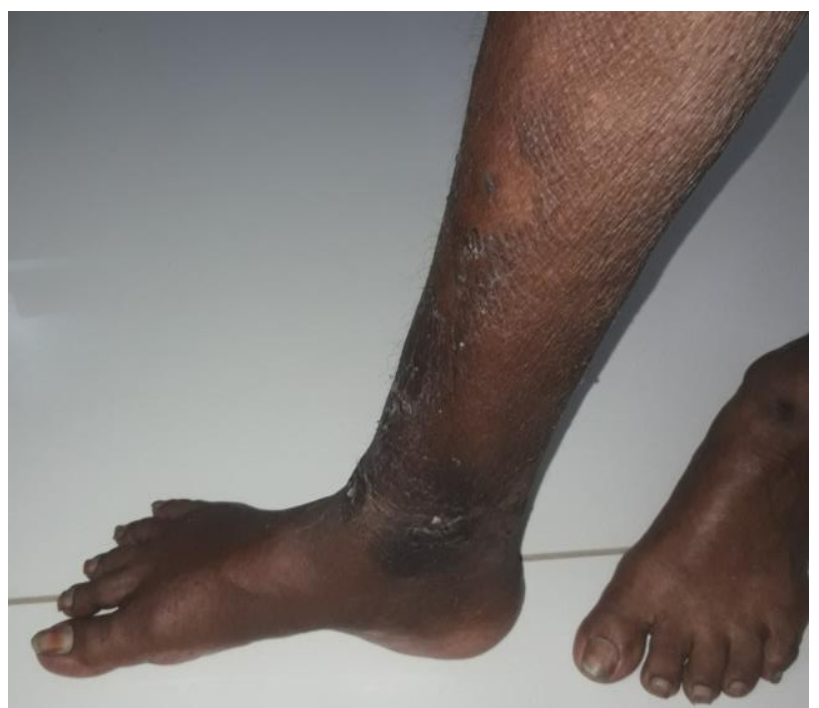

B.

Fig 12: functional out come case 2 


\section{Conclusion}

Ilizarov ring fixator can be considered as safe effective definitive treatment option for paediatric compound \& comminuted tibia fractures as its minimally invasive, biomechanically stable, dynamic, versatile treatment modality which promotes not only fracture union but also allows rapid early rehabilitation and functional recovery, how ever larger sample size \& longer follow up will be needed to prove the same.

\section{Acknowledgments}

Mrs Rekha Vishnu yadkikar, Dr Akanksha ojha, Dr K M ojha, Dr Rahul kunkalol, Dr Ravi Jat, Mr Vikas vishwakarma, Miss Ranu chouhan, Mr Avinash raju, Mr Rajesh thakur They helped in compiling, analysing data and provided technical support .

There was no monitory gains in any form by authors

\section{Refences}

1. Monsell FP, Howells NR, Lawniczak D. High energy open tibial fractures in children treatment with programmed circular external fixator, JBJS, 2012;7:94-B,

2. Shrivastava AK, Mehlman CT. Elastic stable intramedullary nailing of tibial shaft fractures in children, J. Paedtric orthopaedics 2008;28152-158

3. Bahaa Ali Kornah, Hesham Mohamed safwat. External Fixator as primary \& definitive treatment of closed diaphyseal lower limb fractures in children, Ortho \&Rheum open access journal 2017;7(3),MS .ID 555713

4. Sandeep Sriram, Nishant Jajee, Prashant Dhanraj, Functional outcome of tibial fractures in children treated by supracutaneous locking pltes, international journal of orthopaedic sciences 2021;7(1):738-741.

5. Spiegel, Mast JW Internal \& external fixation of fractures in children, Orthop clin north Am 11(3);405421

6. Simon AL, Apostolou N. Paediatric tibial shaft fractures treated by open reduction \& stabilization with monolateral external fixation, journal of children's orthopaedics 2018;12;20-28

7. Norman D, Peskin B, The use of external fixators in the immobilization of paediatric fractures, Arch Orthop trauma surgery 2002;122;379-382

8. Gordon JE, Schoenecker PL, Comparison of monlateral \& circular external fixation of unstable diaphyseal tibia fractures in children, journal of paediatric orthopaedics B2003;12;338-345

9. Lee S-H, Hong J-Y. Factors related to leg length discrepancy after flexible intramedullary nail fixation in paediatric lower extremity fractures, Journal of paediatric orthopaedics B 2015;24;246-250.

10. Tolo VT External fixation in children's fracture, journal of paediatric orthopaedics 1983;3;435-442.

11. Hansen BA, Greiff J, Fractures of tibia in children, Acta orthopaedica scand 1976;47;448-453.

12. Song KM. Sangeorzan Open fractures of tibia in children, Journal of paediatric orthopaedics. 1996;16:627-634.

13. Gougoulias N, Khanna A. Open tibial fractures in paediatric population; systematic review of the literature, Br Med bull 2009;91:75-85.

14. Gareth P Rogers, Hiang B Tan. Complex tibial shaft fractures in children involving distal physis managed with ilizarov method, strategies in trauma \& limb reconstruction 2019, 14(1).

15. Ligier JN, Metaizeau JP. Closed flexible medullary nailing in paediatric traumatology, Children paediatric 1983;24:383-385.

16. Pandya NK, Flexible intramedullary nailing of unstable / open tibia shaft fracture in paediatric population, Journal of paediatric orthopaedics 2016;36(1):s19-s23.

17. Hossein Aslani, Ali Tabrizi. Treatment of open paediatric tibial fractures by external fixation versus flexible intramedullary nailing; comparative study, Arch trauma Res.2013;2(3):108-112.

18. Bartlet CS, Weiner LS. Treatment of type II \& type III open tibia fractures in children, Journal of orthopaedic trauma 1997;11:357- 362. 